\title{
The impact of the length of fluorescent fiber concentrators on the performance of VLC receivers
}

\author{
W. Ali, Z. Ahmed, W. Matthews and S. Collins, Member, IEEE
}

\begin{abstract}
Fluorophore doped plastic optical fibers can be used to create optical concentrators in receivers for visible light communications, that also act as wide field of view filters. Increasing the length of these fibers allows them to collect more of the signal from the transmitter, however, it can also reduce the bandwidth of the fiber. Results are presented from 3 different lengths of fiber which show that the best length of a fluorescent fiber depends upon the data rate. However, a simple calculation results in a length that is a good choice for a range of OOK data rates.
\end{abstract}

Index Terms Fluorescence, fluorescent concentrator, Optical receivers, visible light communications (VLC), optical wireless communications (OWC), Li-Fi.

\section{INTRODUCTION}

$\mathrm{T}$ he capacity of a VLC channel depends upon a combination of channel bandwidth and the signal to noise ratio (SNR) at the receiver output. The SNR of a channel can be increased using an optical concentrator such as a compound parabolic concentrator. However, conservation of etendue means that if these concentrators significantly increase the SNR they also restrict the receiver's field of view. One approach to increasing the SNR of a receiver, without restricting its field of view, is to use a fluorophore doped optical element as a concentrator. Since fluorescence does not conserve etendue this approach can simultaneously achieve a high optical gain and a wide field-ofview [1-3]. Furthermore, the wavelength-selective absorption of the fluorophore means that these optical elements can also function as wide field-of-view optical filters [3].

The various advantages of using fluorescence in optical concentrators means that commercially available fluorescent fibers have been included in several systems $[2,4,5]$. In these systems using longer fibers creates the opportunity to increase the SNR of the receiver by collecting more light from the transmitter. However, in longer fibers the transit time of photons in the fiber can reduce the fibers bandwidth. Previously, it was suggested that the length of a fiber should be chosen so that the $3 \mathrm{~dB}$ bandwidths arising from the transit time and fluorophore's lifetime are equal [2]. However, an equally

Submitted on $21^{\text {st }}$ July 2021.

This work was supported by EPSRC grants EP/R00689X/1 and EP/R005281/1.

Wajahat Ali, Zubair Ahmed, William Matthews and Steve Collins are with Department of Engineering Science, University of Oxford, Oxford OX1 3PJ, plausible criterion would be to equate the maximum transit time and the fluorophore lifetime. Furthermore, both of these plausible criteria are independent of the data rate. The performance of three different lengths of a commercially available fluorescent fiber have therefore been compared at different $\mathrm{OOK}$ data rates. Results suggest that the previously proposed way of determining the length of the fiber works well for lower data rates. However, using shorter fibers improves the performance of the receiver at higher data rates.

The paper is organized as follows. The expected and measured frequency responses of 3 different lengths of fluorescent fibers are described and compared in section II. The equipment used to transmit OOK data to VLC receivers containing one of the 3 different lengths of fiber is then described in Section III. This section also includes the results of data transmission experiments. Finally, section IV contains the conclusions.

\section{FLUOROPHORE B ANDWIDTH AND TRANSIT TIME}

The excited state of a fluorophore typically decays exponentially with a characteristic lifetime, $\tau_{f}$. This creates a frequency response

$$
R_{f l}(f)=\left(1+\left(f / f_{3 d B}\right)^{2}\right)^{-1}
$$

where the $3 \mathrm{~dB}$ frequency $f_{3 d B}$ and the lifetime are related by

$$
f_{3 d B}=1 / 2 \pi \tau_{f} .
$$

If the fluorophore is incorporated into an optical fiber then it may be necessary to consider the impact of the transit time of photons in the fiber. Previously, it has been suggested that the distribution of transit times creates an additional frequency response [2]

$$
R_{t t}(f)=\operatorname{sinc}\left(\tau_{s} \pi f\right)
$$

where, if $\mathrm{L}$ is the length of the fiber, $n_{c o}$, is its refractive index and $\mathrm{c}$ is the speed of light, then

UK. (emails wajahat.ali@eng.ox.ac.uk, zubair.ahmed@eng.ox.ac.uk, william.matthews@univ.ox.ac.uk and steve.collins@eng.ox.ac.uk). 


$$
\tau_{s}=L n_{c o} / c
$$

The fluorescent lifetime and the transit time together mean that the frequency response of a fluorescent fiber is then [2]

$$
R_{f f}(f)=\operatorname{sinc}\left(\tau_{s} \pi f\right) /\left(1+\left(f / f_{3 d B}\right)^{2}\right)
$$

It has then been suggested that a good method of determining the length of the fiber used in a receiver is to equate the $3 \mathrm{~dB}$ bandwidths of (1) and (3) [2]. This approach leads to the conclusion that a good choice of fiber length is

$$
L=3.791 \tau_{f} c / n_{c o}
$$

Alternatively, the length could be chosen to make the maximum transit time (4) equal to the fluorescent lifetime. This approach leads to the conclusion that a good choice for the fiber length is

$$
L=\tau_{f} c / n_{c o}
$$

However, neither of these approaches take into account the data rate or the additional light collected by longer fibers.

The frequency response of a $8 \mathrm{~cm}, 0.5 \mathrm{~mm}$ square long Kuraray (SCSF-81J) fiber was measured using a Network Analyzer (HP-8712) connected to the transmitter and receiver sides of the data transmission experiment setup described in section III. As expected from such a short fiber the measured response was consistent with (1). Furthermore, the measured 3 dB frequency, $117 \mathrm{MHz}$, corresponds to a fluorophore lifetime of $1.36 \mathrm{~ns}$. Since the refractive index of the fibers is 1.59 [6], (6) suggests that a $97 \mathrm{~cm}$ fiber should be used, whilst (7) suggests that the fiber should be $26 \mathrm{~cm}$ long.

Based upon these estimates three lengths of fibers $(16 \mathrm{~cm}$, $30 \mathrm{~cm}$ and $1 \mathrm{~m}$ ) have been characterized. To ensure that they were uniformly illuminated during experiments these fibers were held in a spiral, or part of a spiral. However, to keep bend losses to significantly less than $10 \%$ the minimum bend diameter used was $5 \mathrm{~cm}[6]$. Figure 1 shows that the two shorter fibers have similar $3 \mathrm{~dB}$ frequencies, $117 \mathrm{MHz}$ and $112 \mathrm{MHz}$.

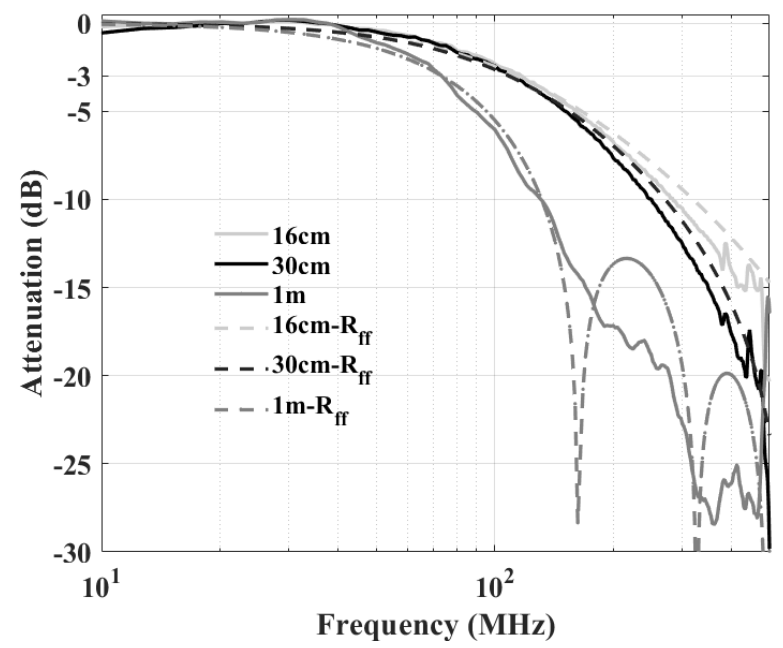

Fig. 1. The measured frequency response of 3 different lengths of fluorescent fiber lengths and their responses calculated using (5).
This figure also shows the fit of (5) to the different sets of measured data. Initially, particularly for the $1 \mathrm{~m}$ fiber, (5) significantly overestimated the fibers responses at higher frequencies. However, (5) implicitly assumes a single mode fiber and the fluorescence fibers are multi-mode fibers. This means that the average path length of light in the fiber will be longer than the length of the fibre. The results in Fig. 1 were calculated assuming a maximum path length that is $15 \%$ longer than the actual length. In addition to explaining the smaller than expected bandwidth the multiple paths between each part of the fiber and the photodetector are believed to attenuate the destructive interference that creates the zeros in (3). These multiple paths would then explain the absence of the expected zeros in the measured frequency response of the $1 \mathrm{~m}$ fiber.

\section{DATA TRANSMISSION RESULTS}

The OOK data rate that can be transmitted across a VLC channel depends upon a combination of the frequency response of the channel and the receiver's SNR. The results in Fig 1 show that the $3 \mathrm{~dB}$ bandwidth of the $1 \mathrm{~m}$ fiber is smaller than the bandwidth of the two shorter fibers. However, at a particular transmitter irradiance this fiber absorbs more light and therefore increases the optical signal reaching the SiPM. For all possible noise sources this will increase the receiver's SNR. The OOK data rates that can be transmitted to receivers containing one of the 3 different lengths of fibers have therefore been investigated.

A schematic diagram of the equipment used to investigate the performance of VLC receivers containing 3 different lengths of fibers in the dark is shown in Fig 2. During these investigations a $10 \mathrm{GHz}$ Tektronix arbitrary waveform generator (AWG) was used to generate OOK pseudorandom binary sequence (PRBS) signals. These signals were then amplified by a Fairview microwave (FMAM3269) amplifier. After amplification a DC bias was added to the amplified signal using a Bias-Tee (ZFBT4R2GW+). The resulting signal was then applied to a Thorlabs L405P20 laser diode (with peak wavelength of $405 \mathrm{~nm}$ ). A polarizer was placed in front of the laser diode so that the transmitter irradiance falling on the receiver could be varied.

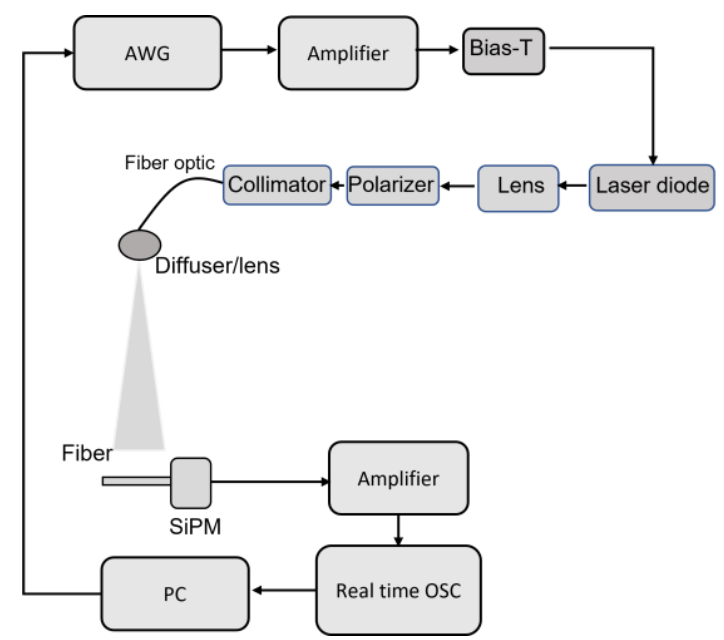

Fig. 2 A schematic diagram of the experiments used to determine the irradiance required to support different OOK data rates at a BER of $10^{-3}$. The distance between the transmitter and the receiver was $30 \mathrm{~cm}$. 
The output light from the polarizer was coupled into a multimode fiber by a fiber collimator. Finally, a diffuser and plano-convex lens were used at the other end of this fiber to create a diffuse beam.

The receiver in these experiments included a silicon photomultiplier (SiPM) coupled to one end of a fluorescent fiber. In particular, the fluorescent fibre was couple to a 30020 J-series SiPM manufactured by ON-Semiconductor [7]. Like all SiPMs the 30020 is an array of microcells, which each contain an APD biased above its breakdown voltage. Each microcell in this SiPM also contains a resistor that stops the otherwise selfsustained, photon-initiated, avalanche event. The voltage change on the circuit node between the resistor and the APD, caused by the detection of a photon, results in a $1.4 \mathrm{~ns}$ wide output pulse. For these experiments these pulses were amplified by a ZFL-1000LN+ amplifier. Once it was amplified the SiPM's output signal was captured by a Tektronix MSO64 (4 $\mathrm{GHz}, 24 \mathrm{GS} / \mathrm{s}$ ) oscilloscope (OSC). Finally, the captured signal was post-processed offline using MATLAB ${ }^{\circledR}$. This postprocessing usually included applying decision feedback equalization (DFE) before determining the resulting bit error rate $(\mathrm{BER})$.

Increasing the length of a fiber both changes its frequency response and increases the signal received by the SiPM at a particular transmitter irradiance. To investigate the impact of the different frequency responses of the fibers the polarizer was used to ensure that the SiPM signal was the same for each fiber. The average irradiance from the transmitter falling on the fiber was then measured using the Newport 818-SL power meter. These results, third column of Table I, show that, because they collect more light from the transmitter, the two longer fibers require a lower transmitter irradiance to generate the same SiPM signal. However, the reduction in irradiance achieved using the $1 \mathrm{~m}$ long fiber is smaller than that would be expected if self-absorption by the fluorophore is negligible. Selfabsorption may therefore also be contributing to the lack of zeros in the measured frequency response of the $1 \mathrm{~m}$ fiber.

The OOK data rates that can be achieved with the same SiPM signal and a BER of $10^{-3}$, with and without DFE, were then measured. Another important observation from Table I is that although the $16 \mathrm{~cm}$ and $30 \mathrm{~cm}$ fibers have similar $\underline{3 \mathrm{~dB}}$ bandwidths the data rates that they support are very different. These results show that at the $3 \mathrm{~dB}$ bandwidth does not contain enough information to determine the performance of a fiber in a receiver.

In other experiments the average transmitter irradiance required to support $\mathrm{OOK}$ data rates, with a BER of $10^{-3}$, after DFE were determined. A representative eye diagram from these

Table I A summary of some key results.

\begin{tabular}{|c|c|c|c|c|}
\hline $\begin{array}{c}\text { Length } \\
(\mathrm{cm})\end{array}$ & $\begin{array}{c}3 \mathrm{~dB} \\
\text { Bandwidth } \\
(\mathrm{MHz})\end{array}$ & $\begin{array}{c}\frac{\text { Transmitter }}{\text { Irradiance }} \\
\left(\mathrm{mWm}^{-2}\right)\end{array}$ & $\begin{array}{c}\text { Data } \\
\text { Rate } \\
\text { DFE } \\
(\mathrm{Mbps})\end{array}$ & $\begin{array}{c}\text { Data } \\
\text { Rate no } \\
\text { DFE } \\
(\mathrm{Mbps})\end{array}$ \\
\hline 16 & 117 & 81.1 & 950 & 445 \\
\hline 30 & 112 & 43.9 & 775 & 395 \\
\hline 100 & 73 & 20.3 & 475 & 200 \\
\hline
\end{tabular}

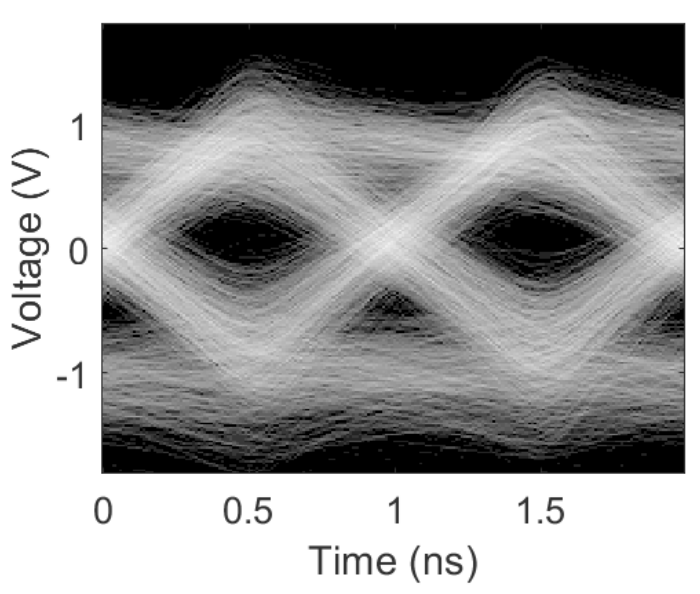

Fig. 3. A typical eye-diagram, in particular the eye diagram at $1 \mathrm{Gbps}$ and a BER of $10^{-3}$ when the $16 \mathrm{~cm}$ fiber is used in the receiver.

experiment in shown in Fig. 3. An important feature of 30020 SiPMs is that whilst a microcell is recharging after an avalanche event its ability to detect another photon is reduced. The most important consequence of this behavior is that the response of the SiPM becomes non-linear at high irradiances [7]. The results in Table I were obtained at the top of the linear range of the SiPM. A comparison of the data rates with DFE in Table I and the results in Fig 4 show that most of the results in Fig. 4 were obtained when the SiPM has a linear response.

The results in Fig 4 show that at the lowest data rate the longest fiber, requires the lowest transmitter irradiance. However, between $250 \mathrm{Mbps}$ and $800 \mathrm{Mbps}$ the $30 \mathrm{~cm}$ fiber requires the lowest irradiance. Finally, at the highest data rates the $16 \mathrm{~cm}$ fiber requires the lowest irradiance. These results show that the best length of a fluorescent fiber depends upon the OOK data rate. However, for this particular fluorescent fiber using a fiber whose length was chosen using (6), i.e. the $1 \mathrm{~m}$ fiber, is a good choice when the target data rate is less than twice the fluorophores bandwidth. For higher data rates a shorter fiber whose length is determined using (7) is a better choice. However, even shorter fibers can be an even better choice at the highest data rates.

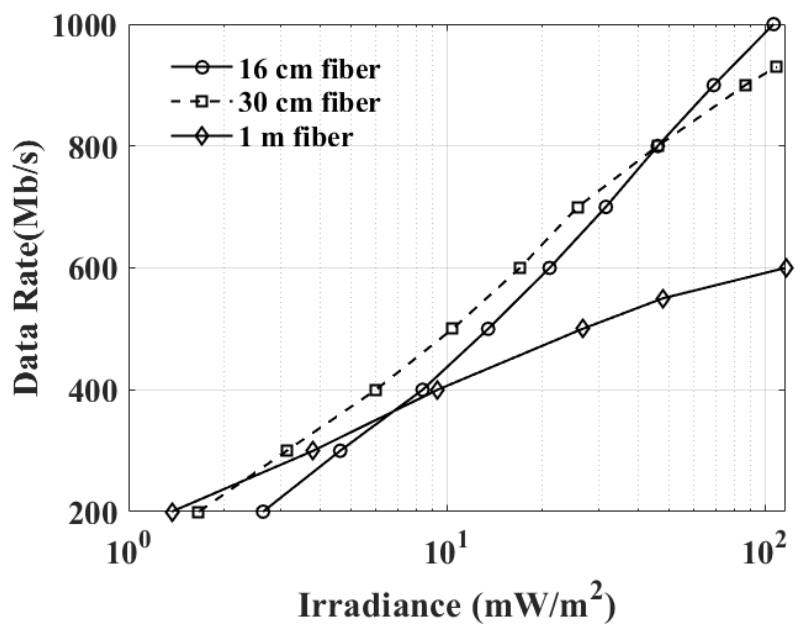

Fig. 4. Data rates with DFE as a function of irradiance for various fiber lengths. 


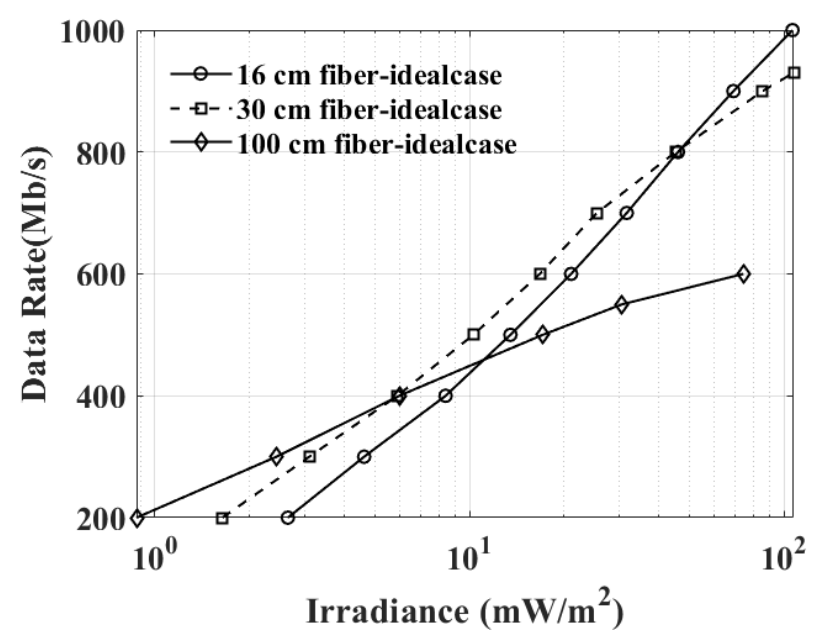

Fig. 5. Data rates with DFE as a function of irradiance for various fiber lengths

The reduction in transmitter irradiances in Table $\mathrm{I}$ as the length of the fiber increases is less than would be expected in an ideal, loss-less fiber. In particular, the $1 \mathrm{~m}$ fiber is 6.25 times longer than the $16 \mathrm{~cm}$ fiber. However, using a $1 \mathrm{~m}$ fiber only reduces the required irradiance by a quarter. This suggests that to support the same data rate an ideal $1 \mathrm{~m}$ long loss-less fiber would only require $64 \%$ of the irradiance shown in Fig 4. Similarly, an ideal $30 \mathrm{~cm}$ fiber would require only $98 \%$ of the irradiance shown in Fig 4.

Fig. 5 shows the predicted performance of ideal lossless fibers obtained by multiplying the irradiances results for the $1 \mathrm{~m}$ fiber in Fig. 4 by 0.64 and the results for the $30 \mathrm{~cm}$ fiber by 0.98. The results in Fig. 5 show that for a loss-less fiber the best length still depends upon the $\mathrm{OOK}$ data rate. However, if a fiber is loss-less then any fiber whose length is determined using (6) will be a good choice for data rates up to 3.5 times the fluorophores bandwidth. At higher data rates a fiber with a length determined by (7) is a good choice, with even shorter fibers being better at higher data rates.

Finally, the differences between the results in Fig. 4 and Fig. 5 show that the choice of the best length of fiber depends upon the losses in the fiber. However, at higher data rates the best choice of length is short enough for losses to be negligible when a SCSF-81J fiber is used. Furthermore, for the highest data rates they are short enough that straight fibers could be integrated into host systems including laptops and tablets to create receivers with a wide FOV [5].

\section{CONCLUSION}

Fluorophore doped optical elements can be used to create wide field of view VLC receivers which concentrate light from the transmitter whilst filtering out ambient light. However, the fluorophore has a lifetime that might limit the bandwidth of the receiver. In addition, if the concentrator is large, the transit time of photons in the concentrator might also limit its frequency response. Previously, it has been suggested that the length of a fluorescent fiber concentrator should be chosen by equating the $3 \mathrm{~dB}$ frequencies arising from the transit time and the fluorophores lifetime [2]. Alternatively, the length of the fiber could be chosen to make the maximum transit time equal to the fluorophore lifetime.

Results have been presented which show that the choice of the fiber length should take into account the required OOK data rate. However, for higher data rates, the fiber whose maximum transit time equals its fluorophores lifetime is a better choice than a fiber whose length is determined by equating $3 \mathrm{~dB}$ bandwidths.

For the SCSF-81J fluorescent fiber this better choice of length, $30 \mathrm{~cm}$, means that self-absorption in the fiber is insignificant. More importantly, it is suitable for integration into host systems including laptops. Furthermore, up to 36 of these fibers can be connected to the SiPM used in these experiments. This suggests that by using a bundle of fibers connected to the SiPM it should be possible to reduce the irradiances shown in Fig. 4 by at least an order of magnitude. In addition to creating this type of receiver, any future work should include an investigation to determine the optimum length for each OOK data rate or when orthogonal frequencydivision multiplexing (OFDM) is used. However, when OOK is used the power penalty required to support DFE means that this investigation is expected to lead to numerical, rather than analytical, results.

\section{REFERENCES}

[1] P. P. Manousiadis et al., "Wide field-of-view fluorescent antenna for visible light communications beyond the étendue limit," Optica, vol. 3, pp. 702-706, 2016.

[2] T. Peyronel et al., "Luminescent detector for free-space optical communication," Optica vol. 3, pp. 787-792, 2016.

[3] Ali, Wajahat, et al. "A Gigabit VLC receiver that incorporates a fluorescent antenna and a SiPM." Journal of Lightwave Technology. doi: 10.1109/JLT.2021.3095398. 2021.

[4] Chun Hong Kang et al., "Ultraviolet-to-blue color-converting scintillatingfibers photoreceiver for 375-nm laser-based underwater wireless optical communication," Opt. Express 27, pp. 30450-30461 2019.

[5] A. Riaz and S. Collins, "A wide field of view VLC receiver for smartphones," 2020 European Conference on Optical Communications (ECOC), 1-4 2020.

[6] Kuraray Co Ltd, "Plastic scintillating fibers", https://www.kuraray.com/uploads/5a717515df6f5/PR0150_psf01.pdf

[7] W. Matthews, Z. Ahmed, W. Ali and S. Collins, "A 3.45 Gigabits/s SiPMBased OOK VLC Receiver," IEEE Photonics Technology Letters, vol. 33, pp. 487-490, 2021. 